\title{
L'AVENIR DE LA PHILOSOPHIE
}

'IL est un point que les réflexions contemporaines sur le temps $\boldsymbol{N}$ ont mis en relief et inscrit profondément dans notre conscience, c'est que l'avenir qu'on espère dépend directement du présent qu'on réalise. Telle est notre attitude en face du présent, telle elle sera inéluctablement en face de l'avenir; et donc l'avenir lui-même, puisque ce dernier n'est rien sinon un présent caché encore, au cœur d'un présent déjà actuel, et explicite. Il est en effet de l'essence même de notre condition humaine d'avoir un présent structuré et comme stratifié. Certes on peut imaginer le présent comme un point sans épaisseur, comme la limite évanescente entre un segment de ligne qui marque le passé à jamais révolu, et l'avenir qui se meut vers nous pour basculer inexorablement vers le non-être en franchissant ce présent. Mais cette image, utile pour bien des tâches, ne correspond d'aucune manière à la réalité vivante de la conscience humaine et des activités qui lui sont propres. Nous sommes dans le présent ce que nous sommes et ce que, en un sens, nous croyons pouvoir dire que nous avons été. Une grande partie de ce que nous appelons le passé, notre passé personnel et sans doute notre passé spécifique, est présent, non seulement à titre de souvenir, pouvant être, ou non, évoqué, mais à titre de schéma dynamique modifiant notre être-au-monde, notre présente réaction au réel le plus réel, à la situation où nous avons à déployer notre activité. Nous sommes présents à notre présent, non pas comme des nouveaux-nés ou des plaques photographiques attendant l'impression sur une surface totalement vierge. Nous sommes dans l'unité (ou la pluralité) de notre être, une présence dynamique au monde, profondément modifiée et modelée et pétrie par la suite ininterrompue des présents par lesquels nous avons fait face à la réalité, avec nos réactions et nos jugements de valeur, nos choix et nos lâchetés, nos ignorances, et les découvertes qui progressivement les ont éliminées, en un mot avec cette durée que Bergson a comparée à la boule de neige, qui en dévalant le long d'une pente ne change pas de substance, mais forme, dans les couches qu'elle retient à chacun des tours 
qui font son mouvement, l'image immobile et comme figée de sa course vers la vallée.

Notre mémoire retient sans doute aussi bien d'autres choses, des images et des scènes et un certain ordre chronologique irréversible entre eux. C'est d'ailleurs à ce genre de contenus, à cette fonction surtout passive et enregistreuse d'objets que nous pensons quand nous nous interrogeons sur l'avenir. Nous projetons la ligne imaginaire du passé avec son aboutissement, le présent, vers un avant imaginaire. Et nous nous demandons en somme si nous retrouverons, fixée sur cette ligne, toujours là, toujours bien accrochée, cette réalité au sujet de laquelle nous interrogeons. La philosophie a glissé le long du temps jusqu'à nous. Elle est là présentement. En regardant vers le passé nous la trouvons présente très loin en arrière. Et nous pouvons nous demander: la philosophie sera-t-elle encore là quand après avoir laissé se dévider une certaine longueur d'avenir vers le passé nous pourrons discerner ce qui se trouve ou non sur la ligne du temps.

On peut ainsi s'interroger sur l'avenir de la philosophic, simplement en se demandant si après une durée plus ou moins longue elle aura continué à venir fidèlement faire partie de notre univers mental. Ceci suppose d'ailleurs qu'on la tient pour déjà présente dans l'avenir, comme possible, et c'est pour cette raison sans doute que d'une manière générale l'attente et surtout le désir confèrent une certaine réalité, justement une réalité présente, dans l'attente et le désir, à cela dont nous voudrions connaître les chances d'un présent effectif après ce présent dans l'attente et le désir.

Mais une telle manière de s'interroger sur l'avenir de la philosophie ou de quelque réalité que ce soit ne tient compte que d'un aspect seulement de la mémoire et du temps. Celui qui nous fait voir à nous-mêmes comme des spectateurs, comme subissant le temps et tout ce qu'il contient. Et, encore une fois, c'est incontestablement là un aspect de notre être au monde. Il est aussi fallacieux et vain de vouloir nous persuader que nous ne sommes que ce que nous décidons d'être par nous-mêmes, et dans un univers qui serait entièrement notre produit, que de vouloir nous réduire au rôle d'un simple passager emporté par un véhicule qu'il ne pilote pas et qu'il n'a pas demandé à occuper, vers des 
destinations inconnues, à travers des contrées qu'il ne peut que contempler avec l'œil indifférent ou étonné du touriste moyen.

A côté du présent qui défile sous nos yeux il y a le présent que nous faisons, plus ou moins consciemment. Un présent qui ne survient pas seulement comme un orage ou une rafale de vent, quelque chose dont on dirait seulement: « il faut passer par là, c'est la vie ", mais le présent qui est fait de nos actions et réactions, de notre manière d'être au monde et non pas seulement de le regarder, et qui modèle en même temps notre propre être au monde et le monde auquel nous faisons face. Se demander alors quel sera l'avenir, c'est se demander non pas: qu'est-ce qui va arriver, comme on attend les résultats d'une compétition sportive à laquelle on ne prend aucune part active, mais: que sera le présent qui vient, du fait que j'aurai agi ou réagi de telle et telle manière dans le présent que je vis, que je façonne dans une mesure plus ou moins large, par ce que je suis actuellement, qui contient tous mes présents dits passés, et qui en fait sont toujours là, à différents niveaux de la conscience, pour façonner mon présent en me façonnant moi-même et ma conduite présente.

En d'autres mots: il y a l'avenir qu'on ne peut qu'attendre, parce qu'il ne dépend pas de nous et dans la mesure où il est indépendant de nous. Il y a l'avenir qui sera fait du présent, demeurant actuel dans les présents qui vont venir, et que nous contribuons à former par notre action présente. L'avenir dépend alors de la manière dont nous vivons le présent. Tel présent, tel avenir. Un présent passivement subi en spectateur plus ou moins passif, donnera un avenir fait de souvenirs qui glisseront avec nous le long de la ligne du temps. Un présent que nous aurons façonné demeurera présent en déterminant activement le présent auquel nous continuerons à être présents d'une manière active et responsable, avec toute la vigilance, le sérieux, la gravité et le sens de notre responsabilité qu'il exigera de nous.

S'interroger sur l'avenir de la philosophie, cela peut donc vouloir signifier: y aura-t-il toujours cette réalité qu'on appelle la philosophie et quels visages nouveaux ou familiers présenterat-elle? Ou bien l'attitude philosophique sera-t-elle toujours, et sous quelle forme, un facteur qui façonne le présent des hommes et qui contribuera utilement à le façonner, ou bien ayant jusqu'ici 


\section{B. GEIGER}

contribué en fait à le façonner devra-t-elle abdiquer et laisser la place à d'autres forces, comme l'âge mythique, aux yeux de Comte, devait céder la place à l'âge métaphysique, et celui-ci à son tour à l'âge scientifique et positif?

Il n'est pas difficile de comprendre que la réponse dépend à la fois de l'idée qu'on se fait de la fonction de la philosophie, et des possibilités qu'on attribue à notre humaine nature par rapport à sa libération à l'égard du passé, c'est-à dire de son expérience historique.

Or, l'un des premiers points qu'il importe de souligner énergiquement si l'on veut poser le problème sur son véritable terrain et ne pas se laisser enfermer dans les limites d'un contexte déterminé, c'est que la philosophie, non plus qu'aucune autre branche de l'enseignement, y compris l'écriture ou le calcul, la géographie et la comptabilité, n'est née pour être une matière d'enseignement. Tout ce qu'on enseigne a été d'abord découverte et réponse à des questions que notre vie, la vie des hommes à travers les siècles, a conduit l'humanité à se poser en vue de fins bien déterminées; fins élémentaires d'abord, comme manger, se vêtir, s'abriter, découvrir et noter les endroits où la nourriture se trouvait avec plus de facilité et d'abondance, etc.

À mesure que les modes de vie changeaient, passant des formes les plus simples et menées par des groupes relativement faibles à des sociétés plus compliquées et plus nombreuses, les problèmes changeaient, avec les possibilités de les résoudre, et de transmettre les résultats acquis par la formation directe, individuelle ou par groupes plus ou moins nombreux. L'enseignement est la manière de transmettre les découvertes faites, mais elle ne peut pas avoir pour but un enseignement pur, c'est-à-dire l'enseignement pour l'enseignement, sans se préoccuper de la raison d'être de ce bagage qu'on traîne à travers les âges, jusqu'au moment où intervient ce qu'on appelle la révision des programmes qui n'est pas autre chose que la prise de conscience de l'inutilité de certaines matières, de certains de leurs éléments, ou de la manière de l'enseigner, qui peut se faire beaucoup plus simplement. Il y a enseignement, parce qu'il y a en fait parmi les hommes cette inégalité: que les uns découvrent et inventent, améliorent des découvertes déjà existantes, font surgir de nouvelles questions et essaient de leur 
mieux d'y répondre, alors que d'autres sont surtout réceptifs, demandent qu'on leur montre comment on s'y prend pour fabriquer un arc ou piloter un avion que d'autres ont construit et mis au point, et que, de toute manière, il y aura toujours l'inégalité de l'âge, les plus jeunes entrant dans un univers déjà façonné par les inventions, les découvertes, le savoir et le savoir-faire, les théories scientifiques ou philosophiques, élaborés par les générations antérieures, vivantes ou disparues.

La philosophie ne fait pas exception à cette règle. Elle a été d'abord, par un événement parfaitement imprévisible, par une sorte de mutation brusque, le fait de certains individus, qui, au lieu de se contenter de questions pratiques et techniques, trop négligées d'ailleurs, par la suite, dans les questions mêmes que les philosophes allaient se poser pendant des siècles, s'interrogèrent sur le sens de l'existence humaine, sociale d'abord semble-t-il, mais aussi et très tôt, personnelle et individuelle, et sur le sens et l'origine de toutes choses. Jaspers a montré, d'une manière qui semble convaincante, que durant trois siècles, exactement les mêmes, cet événement s'est produit, avec des variations importantes d'ailleurs, dans des pays aussi différents et séparés les uns des autres que la Chine, l'Inde, la Perse, la Palestine avec les grands prophètes, la Grèce. Certes il est amené à souligner ce fait par l'importance décisive qu'il attache à l'avènement de la conscience, à ce qu'il appelle les situations limites, cet affrontement de la conscience individuelle, se dressant face à l'univers tout entier et à elle-même pour poser les questions qui n'avaient pas été posées jusque là dans l'histoire de l'humanité ou dans la vie de l'individu: devenir personne, existence, au lieu d'être conduit par la tradition, les coutumes, voire les instincts ou le conditionnement sociologique.

Tout est mis en question, et il semble que le milieu dans lequel vivaient ces premiers philosophes se trouvait, au moins partiellement, en attente des mêmes questions, et attendait des réponses. Certes la philosophie a posé et cru pouvoir résoudre une foule de problèmes qui maintenant ressortissent à des procédés d'investigation d'un type différent: nature de l'univers et sa formation, mouvement des astres et nature de la vie, cosmogonie et même médecine et physiologie. Mais c'est que ces questions relevaient 


\section{B. GEIGER}

sans doute alors de la même interrogation fondamentale: qu'est cet univers dans lequel nous vivons, notre raison d'être, notre véritable destinée, la véritable manière d'être un être humain digne de ce nom? Pour le dire complètement il faudrait en effet tout savoir. Faute de soupçonner toute la complexité des données, les réponses étaient faites d'une manière qui aujourd'hui nous semble enfantine, mais qui était sans doute le commencement nécessaire, les premiers pas qu'il fallait faire pour être en mesure d'aller plus loin.

Quoi qu'il en soit, les réponses essentielles étaient celles qui avaient trait à la raison d'être de l'univers et de notre présence en lui. Réponses que les philosophes, les sages croyaient pouvoir donner mieux que les religions mythiques qui les avaient précédés, et qu'ils communiquaient sous forme, non d'un enseignement de type scolaire, mais plutôt, et pendant des siècles, sous forme de quelque chose qui ressemblerait plutôt à une initiation ou à la direction donnée à ses adeptes par un maître de sagesse.

La philosophie certes a changé au cours des siècles. Elle est devenue tantôt l'effort poursuivi par un penseur pour construire un système où l'ensemble de l'univers et tout ce qui se passe en lui se trouverait mis en place, tantôt réflexion sur les sciences une fois qu'elles se furent libérées de la philosophie et eurent conquis leur autonomie. De nos jours elle est redevenue interrogation des esprits sur l'être humain, ses fins, ses raisons d'être, au moins chez les philosophes de l'existence, les philosophes de l'esprit comme Lavelle ou Le Senne, alors que d'autres limitent leur ambition à l'analyse du langage, à la logique mathématique, ou à la philosophie des sciences.

Qu'elle soit interrogation sur l'ensemble de l'univers, son histoire, celle de l'humanité en particulier ou réflexion plus centrée sur les problèmes de l'individu ou de la personne, la philosophie a été la pointe de la conscience humaine, s'interrogeant sur ce que celle-ci peut et doit entreprendre pour se réaliser aussi pleinement et aussi authentiquement qu'elle le peut, puisque, pour cet aspect de son être, par où elle émerge de l'animalité, c'est-à-dire du biologique et de l'équipement psycho-physiologique, elle possède une certaine liberté de se déterminer elle-même, de faire être par son propre choix telle 
conception de sa fin, de sa raison d'être. Et c'est justement parce que nous ne sommes pas entièrement équipés pour réaliser notre existence, comme les animaux, que nous nous posons des questions au sujet de cet univers dans lequel notre conscience s'est éveillée et de notre propre projet.

Et puisque cet éveil radical de la conscience est toujours et nécessairement un événement, la naissance à une nouvelle manière d'être face au monde et à soi-même, et à leur commun principe, il ne saurait être, par définition, l'effet d'un enseignement passivement subi et qu'il suffirait d'apprendre par cœur. N'est-il pas plutôt comme l'apparition, au milieu des immensités de l'Océan, d'une terre nouvelle, inconnue jusque là? Si la philosophie doit demeurer, parmi les hommes, ce qu'elle a été lors de ses premières manifestations, elle sera, dans des contextes historiques et culturels modifiés, en partie par sa présence, au cours des siècles, le même éveil et la même naissance à un mode de vie plus exigeant et plus lucide. Sinon, elle sera n'importe quoi, et notamment une matière, justement, dont un homme dit cultivé doit avoir entendu parler, qu'il faut en tout cas avoir apprise pour se voir admis à certains examens, et passer pour un homme compétent en une matière appelée philosophic, à moins qu'elle ne soit tout simplement un objet de curiosité pour l'historien, l'anthropologue et, évidemment, pour le psychologue dit des profondeurs.

Non qu'il y ait contradiction entre l'enseignement comme tel et l'éveil de l'esprit philosophique, non plus qu'entre le métier de l'artiste, qui s'apprend, et l'inspiration qui survient comme un don. L'authenticité d'une vocation ne se reconnaît-elle pas justement dans l'heureuse union et l'acceptation loyale des deux composantes indissociables de toute véritable création? Le métier seul ne suffit pas, mais il ne risque d'étouffer l'inspiration qu'au moment où celle-ci a cessé d'être la mesure intérieure de l'œuvre à naître, pour céder la place à d'autres instances moins exigeantes.

Inversement, le rejet de l'enseignement et de la part de docilité qu'il entraîne n'est par le signe infaillible, trop facile, il faut bien l'avouer, d'un éveil à la philosophie et à ses interrogations. Bien des "mises en question» lancées sur le marché philosophique ne sont guère autre chose, tout d'abord, que l'ex- 


\section{B. GEIGER}

pression du besoin normal et nécessaire des jeunes générations, de leur désir naturel et légitime de créer un style de vie dans un univers qui leur soit fraternel, puisqu'il traduirait, et, par là, rendrait possible la manière d'être qui est la leur. Mais il faut bien prendre garde que, sous l'affrontement, en apparence tout négatif, sous le besoin de rupture où le biologique et le psychologique semblent les facteurs décisifs, se prépare aussi et se dessine déjà la mutation, d'où sortira éventuellement, après les tâtonnements inévitables, la manière spécifiquement humaine de conduire son existence par l'acceptation lucide des devoirs et des responsabilités dans l'aventure de l'humanité, que la philosophie, même si elle n'en détient pas l'interprétation ultime, peut et doit contribuer à clarifier.

Cependant la philosophie a aussi son histoire. Ce qui veut dire que sa présence aujourd'hui contient ou du moins doit contenir, chez celui qui en connaît le passé, les présents qu'ont été au cours du temps les différentes philosophies. Les philosophies sont les publications de la philosophie disait Le Senne. Formule ambiguë, puisqu'elle semble rattacher les philosophies réelles des philosophes à quelque philosophie en soi, aussi abstraite que l'Histoire (avec majuscule) qui est censée imprimer son sens à elle, non seulement à l'histoire réelle, mais encore aux prophètes de l'histoire à qui elle a révélé son sens et qui se sentent portés par elle dans la seule direction vraie.

Faisons abstraction de cette abstraction. Il reste alors le fait indéniable que, depuis ses lointaines origines, la philosophie n'a cessé de se transformer. Quand l'effondrement de la culture antique eut interrompu la tradition pour un temps, brisant la continuité de la conscience philosophique et de ses interrogations, elle se reforma, non sans peine, à partir du moyen âge, dans un univers mental bien différent. En dépit des efforts pour briser cette continuité, pour découvrir et déterminer pour la philosophie un point de départ, comme le cogito, qui ne devrait rien au passé, donc à l'histoire, nous savons que nous vivons toujours, en Occident, d'une même tradition. Non pas une tradition linéaire, non pas méditation d'un problème déterminé dont la solution aurait enfin été trouvée, à la manière dont Aristote nous présente l'histoire de la philosophie au début de sa métaphysique. 
Mais plutôt voyage d'exploration et de découverte, dont la direction demeure constante, dont le paysage ne cesse de se transformer à mesure que le voyage se déroule.

L'image cependant n'est exacte qu'en partie. Elle est même dangereuse, puisqu'elle implique justement cette erreur essentielle de perspective, d'un être humain demeurant identique et impassible en présence d'un spectacle qu'il ne ferait qu'enregistrer comme le spectateur devant le petit ou le grand écran voit défiler des images. En réalité le spectateur détermine le spectacle en grande partie, puisqu'il lui donne son sens à partir du sens qu'il a ou se donne à lui-même, face au monde ou dans le monde dont il est tout ensemble le principe de surgissement par le sens ou le projet d'être qu'il est lui-même, et le reflet. Car le monde n'est pas, quoi qu'on veuille nous faire croire, en tout ce qu'il est, le corrélat rigoureux de notre projet, encore qu'il contienne de toute évidence de quoi lui répondre, le soutenir, le faire aboutir par l'action ininterrompue sur lui, aspect essentiel de notre existence, et qui n'aurait aucun sens si le monde était purement et simplement le reflet docile de notre projet.

Pour la philosophie ceci revient à dire que par la question qu'elle a posée tout d'abord au sujet de l'univers en sa totalité, de sa raison d'être, de notre destinée en rapport avec cet univers où nous savons que nous avons pris forme un jour et où notre corps va se dissoudre à nouveau après un temps plus ou moins long, les termes de l'interrogation n'ont cessé de se modifier. Mouvement en extension, plus facile à constater, dans le sens de l'infiniment grand et de l'infiniment petit, dans la durée et tout ce qu'elle suggère de profondeur d'histoire, de devenir et de destruction, de naissances et de morts, de création et de catastrophes accumulées derrière nous.

Mouvement en profondeur, plus facile à ignorer ou à méconnaître, qui correspond à ce que nous appelons les prises de conscience, les mises au jour de zones de notre propre être, par des actualisations ou des mutations, aussi radicales que celles qui séparent le nourrisson de l'homme adulte. Histoire de cette conscience, de ces ascensions que chacun doit refaire, qu'il peut manquer, que l'histoire peut manquer à son tour, dont aucune finalement ne s'impose avec la nécessité d'un déterminisme 


\section{B. GEIGER}

physique ou biologique, et qui nous laissent donc libres et responsables de les accueillir, de les faire être nôtres ou de les écarter.

Si la philosophie doit demeurer cette interrogation radicale, si d'autre part, le sujet qui interroge doit refaire réellement l'essentiel de cet itinéraire des mutations ou des âges de l'homme, auquel la psychologie, voire la psychanalyse restitue son importance décisive pour le développement normal de la personnalité, elle ne peut pas consister seulement en la transmission d'un savoir, en la répétition de thèses ou de solutions, si excellentes soient-elles en elles-mêmes, pour un esprit qui peut les comprendre et les assimiler. Elle ne suit pas, et n'a jamais pu suivre, en dépit de toutes les objurgations de Kant, la voie large ou la grande route qui enfin permet d'aller droit au but et de laisser le passé derrière soi. Sur la ligne du temps, où bien d'autres formes de notre activité déposent les réussites dont chacune rend plus ou moins inutiles les précédentes, la philosophie, dans la mesure où elle veut être une interrogation du tout de l'homme, suit un mouvement plus compliqué, mouvement cycloïdal; car chaque individu recommence l'aventure par son début, physiologiquement d'abord, comme unicellulaire, puis parasite, avant d'affronter l'existence indépendante du nouveau-né et parcourir le cycle des stades de la vie, avec leur qualité, leur mode typique de nous faire affronter l'univers des hommes et l'univers' tout court, et chercher à la même question essentielle, dans un univers transformé et en continuel changement, la réponse, dont il est tout surpris de découvrir qu'elle avait reçu, par d'autres pèlerins partis avant lui, un sens si profondément semblable au sien que le sens de sa propre aventure, de sa raison d'être et de son aboutissement s'en trouvent transposés et comme illuminés d'une lumière nouvelle.

Je ne veux pas du tout dire par là que la philosophie ne puisse se muer aussi en réflexion critique sur les sciences, c'est-à-dire sur une activité importante, mais particulière de l'être humain; ou bien en positivisme logique, en analyse du langage, ou bien en l'effort de présenter un système cohérent de l'univers et de tout ce qu'il contient. Je veux simplement dire que la philosophie est aussi, et a été d'abord cette grande interrogation que l'homme a posée au sujet de l'aventure de l'univers, de son origine et de 
sa destination, au sujet de sa propre existence, de son origine et de sa destination, et ceci, à partir d'une mutation de l'esprit humain lui-même, surgissant dans son autonomie, dans la conscience lucide de sa propre réalité si étrangement indépendante de l'univers, où tout semble lié à tout, dans une solidarité et une absence de conscience dont seul l'état de l'embryon dans le sein maternel peut nous suggérer l'équivalent dans notre existence humaine.

C'est à l'intérieur de ce projet radical et peut-être excessif qu'il faut avant tout comprendre la philosophie, et qu'on pourra de surcroît éclairer l'histoire de ses variations, de ses avances et de ses reculs, de ses fixations et de ses libérations. Sinon on identifiera ce projet radical avec l'une ou l'autre de ses spécialisations; mais ces dernières n'ont de sens qu'à l'intérieur de ce projet total dont elles tirent toute leur substance et le prestige qui les entoure. Ce projet, on le voit partout présent où il est question de philosophie. Il anime l'empiriste tout autant que l'idéaliste, le positiviste comme le partisan de la métaphysique spéculative. C'est lui qui fait parler au nom de l'être des penseurs aussi opposés que Sartre et Heidegger. Aucun de ceux qui revendiquent le titre de philosophe n'a jamais consenti à passer pour un simple narrateur, qui décrit ce qu'il voit, ce qu'il y a en fait, ici ou là, à tel moment ou à tel autre, qui nous fait part de ses impressions ou de ses opinions. Tous, au contraire, entendent bien revêtir leur message de l'éclat et du prestige du dé-voilement de ce qui réellement est, du sens, de la valeur, de l'origine et de la fin de l'univers et de notre propre existence.

Il serait tentant certes, et combien rassurant, de voir l'avenir de la philosophie comme celui de notre activité technicienne, où les dernières découvertes sont censées rejeter dans un passé à jamais révolu les trouvailles d'hier et d'avant hier.

Le temps de la philosophie n'est donc pas le temps des créateurs de la mode, ni même celui de la prospective, de la planification sociale, économique ou biologique. Gabriel Marcel a raison de souligner l'espèce d'absurdité qu'il y a, dans le domaine de la philosophie comme de l'art d'ailleurs, de la grandeur morale, de la religion et de la sainteté, de prétendre distribuer la valeur en fonction de la proximité à l'égard du présent, entendu comme 


\section{B. GEIGER}

le dernier cri. Le temps de la philosophie garde dans son présent, ou du moins devrait garder dans son présent, bien des conquêtes que l'histoire événementielle situe dans un passé plus ou moins lointain. Car ces conquêtes ne sont pas simplement des énoncés, elles sont des possibilités d'être à différents niveaux de hauteur ou de profondeur, la mise au jour de zones de notre être humain qui ne sont jamais données comme la couleur de notre teint ou le timbre de notre voix. Ces possibilités de notre être doivent être reconquises, faire l'option d'un nouveau choix pour chaque génération. C'est pour cela que le passé de la philosophie est en grande partie son avenir, non pas un avenir de répétition machinale, de routine, mais cette répétition dont parlait Kierkegaard, dans un contexte différent il est vrai; aussi nouveau, chaque fois, que le regard de l'adolescent, quand il rencontre pour la première fois le visage aimé, dont la nouveauté ne perd rien à ses yeux par le fait que d'autres, innombrables, autour de lui et avant lui, sont favorisés du même émerveillement.

À la question relative à l'avenir de la philosophie il faut donc répondre d'abord ceci: de la philosophie, il y en aura toujours. Elle est d'ailleurs partout, sous une forme ou une autre. Nos petits et grands politiciens et apprentis sorciers, nos romanciers, chansonniers et poètes, nos journalistes et directeurs de programmes de radio ou de TV, l'homme de la rue et le savant, tous ont leur philosophie; les uns la gardent pour leur usage privé, d'autres se font les propagandistes d'autant plus assurés qu'ils ont moins réfléchi, et prennent leurs humeurs pour des intuitions métaphysiques. Ce n'est donc pas la philosophie comme telle qui peut être inquiète sur son avenir. Ce qu'il faut craindre c'est que le métier se perde, ici comme ailleurs, c'est-à-dire la gravité devant l'enjeu, la maturité que seule peut donner la conscience de la responsabilité présente, mais aussi de l'aide que doit apporter la connaissance des luttes et des victoires passées, des conquêtes déjà réalisées, des possibilités offertes à l'homme, non pas seulement pour l'exploration des espaces matériels, mais des profondeurs ou des hauteurs de sa propre conscience et de ses engagements face aux valeurs.

On peut choisir la platitude, le nivellement utilitaire d'un style de vie qui se retrouve ou peut se trouver partout et à toutes 
les époques: le choix pour la primauté de l'utile et du plaisir, par la fuite devant la transcendance, la valeur et la liberté par laquelle nous pouvons nous faire hommage à leur vérité. Mais il faut avoir le courage et l'honnêteté de reconnaître que ce n'est qu'une des possibilités offertes à notre choix d'hommes, qu'elle a été reconnue, acceptée ou rejetée depuis toujours quand l'individu ou une époque ont décidé de leur « être au monde », qu'elle n'est donc pas la seule, enfin heureusement découverte après un progrès dont nous serions l'aboutissement et comme la réussite.

Si la philosophie veut suivre la mode pour s'assurer un présent et par là l'espoir d'un avenir, au lieu d'être le rappel, importun peut-être pour beaucoup, de la totalité des possibles, présents réellement comme nos possibles ou nos possibilités, soit à titre d'appels et d'aspirations scellées encore dans la conscience personnelle, soit comme des possibilités déjà découvertes et réalisées par d'autres, exprimées par eux et déposées dans ce présent de l'humanité qu'est son histoire, elle n'est plus qu'un vain mot, un mensonge et une trahison. Nous devons savoir, et au fond nous savons plus ou moins clairement, que «l'homme passe l'homme ", que chacun d'entre nous enferme des profondeurs, que d'autres avant nous ont explorées en partie et qui appartiennent à ce que nous savons déjà au sujet de nous-mêmes, dont le fait religieux et l'attente de la Parole de Dieu représentent les régions ultimes offertes à la libre acceptation de notre conscience la plus personnelle et la plus vraie. La philosophie risque toujours d'échanger pour le rôle facile, mais combien attristant, d'amuseur distingué, celui qui lui revient de par sa nature véritable, par Celui-là donc vers lequel elle est chargée par lui de nous mettre en route.

Passons rapidement sur la question de savoir s'il y aura toujours un enseignement de la philosophie. On peut toujours mettre n'importe quoi au programme obligatoire des collèges et des universités. Du moment que la matière est obligatoire, on est sûr, aussi longtemps qu'elle le sera, d'un avenir scolaire de la philosophie, d'un métier assuré et même de la vente régulière des manuels et des textes qui figurent au programme. Source non négligeable de stabilité, au moins provisoire.

Ce qu'il faudra craindre alors pour l'avenir de la philosophie, 


\section{B. GEIGER}

c'est que cette dernière se survive comme ces belles qui s'efforcent de conserver sous un masque une apparence de modernité, c'està-dire de jeunesse, promenant le long des ans le visage qui avait peut-être été le leur dans le passé, le visage vivant de leur présent d'autrefois. S'il rencontre encore quelque succès, les hommages vont donc à l'habileté présente du maquillage et au passé d'une jeunesse à jamais révolue. La philosophie elle aussi peut se maquiller, c'est-à-dire procéder à cet embaumement, qu'est tout maquillage excessif, appelé par la peur de la vie et de ses échéances. On peut, sinon l'embaumer, du moins la mettre dans un état de vie au ralenti, en la déposant dans des programmes obligatoires, parmi les choses qu'il faut savoir. Le métier de professeur de philosophie commence à être assez bien payé ici, et c'est une chose souhaitable. Espérons que nous ne verrons pas se former cette philosophie alimentaire, qui, sous prétexte d'être à jour, d'être la plus présente au dernier cri de la mode philosophique, confond le travail authentique des philosophes, avec le battage et la publicité dont toute activité humaine aujourd'hui semble devoir s'entourer pour des raisons évidentes, mais dont la valeur créatrice est souvent proche du zéro absolu.

Plus sérieuse serait la question de savoir s'il faut ou non enseigner une philosophie, laquelle, à partir de quel âge mental. Non seulement tous les esprits ne sont pas curieux de philosophie théorique, mais tous les problèmes ne sont pas également accessibles, d'une manière réelle et vivante, à n'importe quel âge. Aristote a parlé de l'âge où l'on s'intéresse surtout à la logique et à la dialectique; de l'expérience nécessaire pour comprendre de la manière qui convient, les questions de la morale. Saint Thomas a affirmé que les philosophes eux-mêmes ont réservé pour la fin de leur vie les questions métaphysiques, et notamment les problèmes relatifs à Dieu, puisqu'elles supposent une sagesse dont nous ne sommes pas capables à n'importe quel moment de notre vie.

En particulier, nous ne sommes pas mûrs, n'importe quand et n'importe comment, de saisir la vraie nature des questions ou des problèmes philosophiques, leur irritante permanence, le type tout particulier de réponses qu'elles appellent, et cette nécessité surtout de recourir au passé, souvent au passé le plus lointain, 
comme celui des présocratiques, au gré de Heidegger, pour comprendre le sens même des interrogations qui sont les nôtres aujourd'hui, la forme particulière qu'elles revêtent, pour des raisons que le présent immédiat ne permet pas de saisir, un peu comme la psychologie des profondeurs affirme que les angoisses ou les phobies du malade qui lui semblent devoir s'expliquer par des dangers présents ou futurs, ne sont au vrai que la rumination d'un passé non résorbé et toujours présent, en raison justement de ce défaut d'assimilation consciente.

Un passé en effet contre lequel le sujet est en réaction, surtout si ces réactions comportent des motifs mal éclairés, peut empêcher et empêche en fait l'assimilation normale du passé en ce qu'il devrait avoir de fécond, diversement d'ailleurs aux différents niveaux de la conscience. Nous assistons aujourd'hui, un peu partout, à la crise de croissance, ou aux conflits des générations. Le problème de l'attitude à adopter en face de la philosophie n'en est, pour une bonne part, qu'un aspect particulier. Ce conflit tient sans doute à l'accélération de l'histoire, aux changements rapides de l'univers que nous façonnons, et qui interdit aux jeunes de reconnaître celui dans lequel ils vont entrer comme pouvant et devant simplement prolonger celui de leurs anciens. Les affirmations de ceux-ci touchant cette accélération devraient d'ailleurs leur interdire de prétendre simplement reconduire, par un apprentissage docile de leurs propres vues et comportements, imposé aux jeunes, leur univers dont ils devraient pouvoir admettre lucidement qu'il sera en partie dépassé. Le problème de la philosophie, d'une philosophie présente à l'univers réel tel que nous le vivons reçoit de ce fait un éclairage dont il serait vain de vouloir nier l'importance. Tout le problème est justement de savoir dans quelle mesure les problèmes et les questions de la philosophie devraient être affectés par les changements auxquels nous assistons dans la même mesure que la science ou la technique, la mode ou les coutumes alimentaires. Si les questions philosophiques ont un statut et un rythme qui leur sont propres, il faudrait pouvoir montrer concrètement comment et pourquoi. Mais c'est tout un programme qui est ici touché, et qui demanderait un long exposé, après une longue réflexion: celui de l'historicité de la philosophie. Réflexion qui risque d'être 


\section{B. GEIGER}

d'autant plus agissante qu'on prétend davantage en être indemne.

Ce que je voudrais signaler, d'un point de vue plus pratique, c'est que dans la mesure où il existe un passé mal assimile, mettons pour parler concrètement, le thomisme et son mode d'enseignement durant de longues années, ici au Québec, il y a un phénomène d'indigestion qui vient compliquer le problème déjà très complexe de la croissance normale en philosophie et de la présence du philosophe au présent de la condition humaine. L'indigestion aussi est une présence, mais la présence, aujourd'hui, au repas d'hier ou d'avant hier, et qui empêche justement d'être présent au repas d'aujourd'hui. Il faut laisser à l'organisme le temps de se libérer, de rejeter ce qui ne pouvait être assimilé. Temps salutaire, à condition qu'il soit ce qu'il doit être, une libération en vue de tâches positives, en vue de la vie à reprendre, tout en tenant compte, s'il y a lieu, de l'expérience du passé. Un vivant normalement ne se complaît pas dans la restitution, dans la rumination stérile et agressive du passé. S'il le fait c'est qu'il est sur le chemin de l'indigestion névrotique, du refus pour le refus, de la fuite devant le passé, qui serait le commencement de la sagesse si elle était la fuite vers le positif, et non la fuite en avant, ccmme on dit, c'est-à-dire une fuite devant le passé mais sans but, comme un animal saisi de panique et qui se livre à la méthode archaique des essais et des erreurs, laissant à la chance le soin de la réussite; fuite présente devant le passé à travers le présent réel que la conscience ne peut donc pas affronter.

Aucune vie n'est possible, aucune vie n'est vie, si elle ne sait allier, dans l'unité efficace de la présence la plus vigilante, le présent qu'elle doit réaliser et qui est son avenir, au passé, sur lequel elle prend appui. Synthèse difficile, diversement équilibrée, par conséquent diversement à concevoir, selon les niveaux de notre conscience et des tâches qu'elle nous impose.

La vie philosophique est l'une des plus difficiles et des plus hautes, une des plus diversement conçues aussi, et ce sont justement les philosophies. Il n'existe pas pour elle, non plus que pour n'importe quelle autre forme de vie, effectivement vivante, et non pas préalablement mise en condition, ou congelée, d'avenir préfabriqué. Elle est et sera ce que les philosophes en feront, 
encore qu'ils n'empêcheront jamais la philosophie d'avoir ses exigences propres qu'elle saura faire valoir, après des temps d'éclipse plus ou moins longs, contre les efforts de ceux qui croient pouvoir en faire ce qui leur plaît. Les mots se vengent, aimait à dire Brice Parain, et ils nous remettent dans l'ordre un jour ou l'autre, plus ou moins brutalement. L'avenir de la philosophie est donc une question non pas à propos de la philosophie, comme le programme d'une réjouissance ou d'une campagne de charité. Elle est la philosophie elle-même et c'est pourquoi il est aussi vain de s'interroger sur l'avenir de la philosophie, que de consulter le journal sur la météo, mais pour des raisons inverses. Il est vain aussi de vouloir jouer au prophète, puisque de toute manière, qu'on soit optimiste ou pessimiste, qu'on trace tel programme ou tel autre, on est totalement incapable d'en assurer la réalisation, puisque on ne sera présent que par des prophéties et tout au plus par des programmes qu'on aura contribué à élaborer, et que l'on sait clairement, et qu'on veut lucidement que la philosophie demeure, si elle restera présente, affaire de pensée vivante, personnelle, essentiellement; la réponse donc d'un esprit vivant à des questions vivantes, comme il en va dans tous les domaines de la vie authentique de l'esprit.

La philosophie, son enseignement et sa vie, aura l'avenir qu'elle mérite, l'avenir qu'elle fera en étant présente au présent des hommes, non seulement pour le regarder de haut, ou pour le ruminer après coup, mais en assumant les risques de la présence, ses incertitudes et ses tâtonnements, qui sont ceux-là même de la vie. A vouloir les récuser, à vouloir se donner un savoir absolu et intemporel, on peut réussir à se faire illusion à soi-même et aux autres, on n'évite ni l'usure des systèmes dont l'histoire porte témoignage, ni ces traditions pseudo-constantes, qui comme par miracle se métamorphosent, en suivant après coup les vicissitudes de la pensée, dont elles ont toujours possédé d'avance, sous une forme il est vrai discernable uniquement pour les initiés, les vérités prétendues nouvelles.

Mais si la philosophie n'est pas simplement la somme des autres activités humaines, si elle représente une tâche propre et nécessaire, encore qu'accessible à une minorité seulement, dont 


\section{B. GEIGER}

le rayonnement, on le voit, finit par rejoindre les zones les plus périphériques de la conscience commune, son présent ne sera et ne pourra jamais être la présence seulement aux autres formes de l'activité ou de la conscience humaine. Elle a sa tâche propre, qui se révèle à ceux qui ont le recul nécessaire. Elle a donc son présent qu'elle ne doit pas manquer, présent qui est la seule garantie de son authentique avenir, et qui en raison de sa nature propre, lui impose des liens si caractéristiques avec son propre passé, en même temps qu'il doit la rendre présente, de la présence la plus vivante, c'est-à-dire créatrice, orientée vers l'avenir à faire venir, au présent des hommes, nos contemporains, nos frères les plus proches.

En terminant, ce que je puis sans doute faire de mieux, c'est de redire ce que Heidegger a exprimé en des termes particulièrement heureux, au début même de Sein und Zeit (p. I9), où il se propose entre autres choses, de montrer avec la liaison essentielle du temps et de l'être la nécessité de défaire la métaphysique formée par deux millénaires de philosophie, propos révolutionnaires, s'il en fut: «Puisque l'être ne peut être saisi qu'en regard du temps, la réponse à la question de l'être ne peut se trouver dans une proposition aveugle et isolée. La réponse n'est pas comprise quand on a répété littéralement ce que la proposition énonce, ... comme un point de vue qui, peut-être, diverge de la manière habituelle. Que la réponse soit neuve, cela n'a point d'intérêt, et demeure un trait purement extrinsèque. Ce qu'elle contient de positif doit tenir à ceci: qu'elle soit assez ancienne pour nous permettre de comprendre les possibilités préparées par les Anciens».

Université de Fribourg, Suisse

\section{B. GeIGER}

